\title{
An adolescent with idiopathic pleuroparenchymal fibroelastosis. Case report
}

\author{
Emine Atag ${ }^{a}$, M.D., Nilay Bas Ikizoglua, M.D., Yasemin Gokdemir ${ }^{a}$, Associate Ph.D., \\ Ela Erdem Eralp ${ }^{a}$, Associate Ph.D., Gursu Kiyan ${ }^{b}$, Ph.D., Dilek Yilmazbayhan ${ }^{c}$, Ph.D. and Bulent Karadag , Ph.D.
}

\begin{abstract}
Idiopathic pleuroparenchymal fibroelastosis (IPPFE) is a rare disorder recently included in rare idiopathic interstitial pneumonias according to the updated American Thoracic Society / European Respiratory Society classification. IPPFE is characterized by pleural and subpleural parenchymal fibrosis causing volume loss predominantly in the upper lung lobes. Age of onset is variable, IPPFE mainly occurs in third and fourth decades.

We present a 16 year old patient with a 2-year history of exertional dyspnea, nonproductive cough and weight loss. On physical examination, auscultation revealed diminished breath sounds on the upper lobes.Chest radiograph showed apical pleural thickening and volume loss. Computerized tomographic scan (CT) of chest revealed ground glass densities and tubular bronchiectasis predominantly in upper lobes bilaterally, with interlobular septal thickening of the pleura and enlarged mediastinal lymph nodes. Thoracoscopiclung biopsy was performed and histological evaluation showed subpleural fibrosis and elastic staining demonstrated fragmented elastic fiber deposition in the subpleural area and adjacent pulmonary parenchyma suggesting IPPFE. To our knowledge this is the first case in childhood. Therefore, pediatricians should be aware of this disease for the diagnosis and appropriate management. Key words: child, intersititial lung disease, fibrosis.
\end{abstract}

http:/ / dx.doi.org/10.5546/ aap.2018.eng.e147

To cite: Atag E, Bas Ikizoglu N, Gokdemir Yet al. An adolescent with idiopathic pleuroparenchymal fibroelastosis. Case report. Arch Argent Pediatr 2018;116(1):e147-e150. a. Marmara University School of Medicine, Department of Pediatric Pulmonology, Istanbul; Turkey.

b. Marmara University School of Medicine, Department of Pediatric Surgery, Istanbul; Turkey.

c. Istanbul University Faculty of Medicine, Department of Pathology, Istanbul, Turkey.

\section{E-mail address:}

Emine Atag, M.D.: emineatag@gmail.com

Funding: None.

Conflict of interest: None.

Received: 7-7-2017

Accepted: 8-30-2017

\section{Glossary}

IPPFE: Idiopathic pleuroparenchymal fibroelastosis.

FVC: Forced vital capacity.

FEV1: Forced expiratory volume in 1 second

TLC: Total lung capacity.

DLCO: Carbon monoxide diffusion capacity.

$\mathrm{pCO}_{2}$ : Partial pressure of carbondioxide.

CT: $\quad$ Computerized tomographic scan.

RV: $\quad$ Residual volume.

\section{INTRODUCTION}

Idiopathic pleuroparenchymal fibroelastosis (IPPFE) is a rare disorder recently included in rare idiopathic interstitial pneumonias according to the updated American Thoracic Society / European Respiratory Society classification. ${ }^{1}$ Amitani et al. described this entity as upper lobe fibrosis at first, afterwards renamed as IPPFE by Frankel. ${ }^{2,3}$ IPPFE is characterized by pleural and subpleural parenchymal fibrosis causing volume loss predominantly in the upper lung lobes. Age of onset is variable, IPPFE mainly occurs in third and fourth decades and in nonsmokers. Although the etiology is unknown, bone marrow and lung transplantation, chemotherapy, radiotherapy and recurrent infections are risk factors for IPPFE. Genetic and autoimmune predisposition have also been reported. Common symptoms are weight loss, exertional dyspnea, dry cough and, pleuritic chest pain due to pneumothorax. ${ }^{4}$

Hereby, we present a 16 year old patient with IPPFE, to our knowledge this is the first case in childhood.

\section{CASE REPORT}

A 16-year-old male was referred to our outpatient clinic with a 2-year history of exertional dyspnea, nonproductive cough and weight loss. He had received treatment for asthma and suspected tuberculosis. He was non-smoker and did not have any exposure to cigarette smoke. His cousin died due to an undiagnosed lung disorder. He had a low body mass index of $14 \mathrm{~kg} / \mathrm{m}^{2}$. On physical examination, he had flattened chest. Auscultation revealed diminished breath sounds 
on the upper lobes. His oxygensaturation rate was 94\%. Pulmonary function tests revealed severe restrictive pattern with FVC 30\%, FEV1 32\%, FEV1/ FVC: $109 \%$ of predicted. In body plethysmography, markedly reduced total lung capacity (TLC) of $45 \%$ of predicted and carbon monoxide diffusion capacity(DLCO) $34 \%$ of predicted value were obtained. Capillary blood gas measurements revealed hypercapnia $\left(\mathrm{pCO}_{2}: 55 \mathrm{mmHg}\right)$. Autoantibody panel and serological tests were negative for rheumatological diseases. Fiberoptic bronchoscopy and bronchoalveolar lavage fluid analysis were normal. Chest radiograph showed apical pleural thickening and volume

FIGURE 1. Chest radiograph of the patient

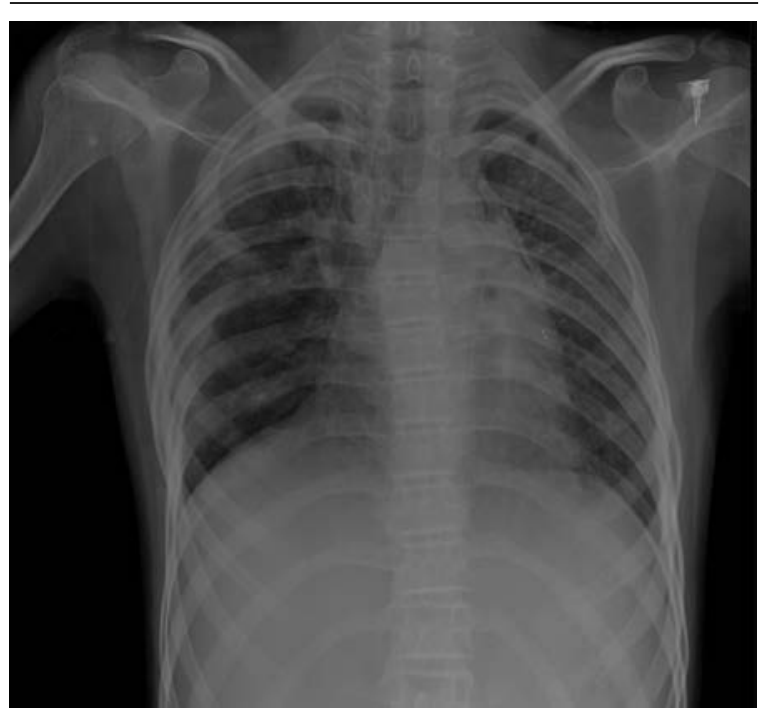

FIGURE 2. Computerized tomographic scan of chest

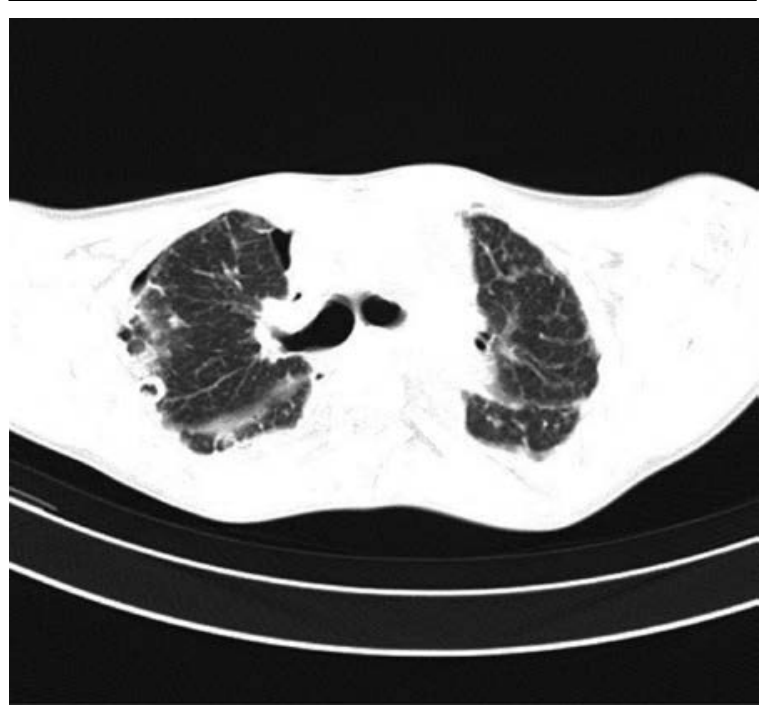

loss (Figure 1). Computerized tomographic scan (CT) of chest revealed ground glass densities and tubular bronchiectasis predominantly in upper lobes bilaterally, with interlobular septal thickening of the pleura and enlarged mediastinal lymph nodes (Figure 2). Thoracoscopic lung biopsy was performed and histological evaluation showed subpleural fibrosis and elastic staining demonstrated fragmented elastic fiber deposition in the subpleural area and adjacent pulmonary parenchyma (Figure 3, Figure 4). Postoperatively pneumothorax occurred at the ipsilateral side, and a chest tube was inserted.

Steroid therapy was commenced and continued for 3 months, but unfortunately he did not get any benefit. Then we decided to start pirfenidone, and lung transplantation was considered, but the family refused both of them, continued steroid. The patient, he died one month later.

Figure 3. Subplevral fibrosis, 21073-14, elastica van gieson stain, $x 40$

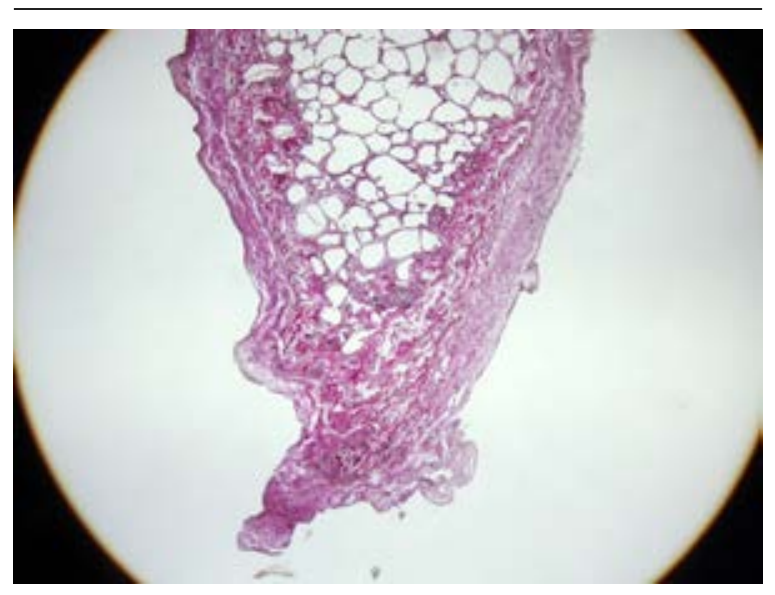

FIGURE 4. Fragmanted elastic fibers, 21073-14, elastica van gieson stain, $x 400$

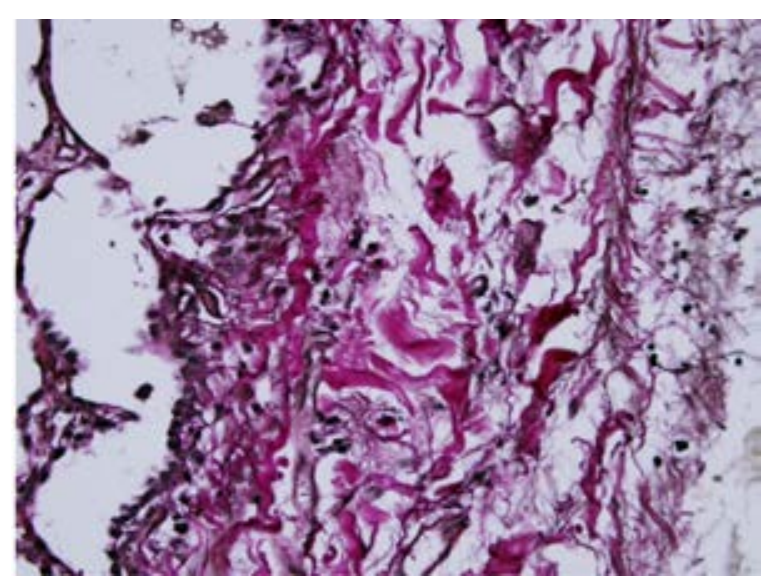




\section{DISCUSSION}

IPPFE is a form of interstitial lung disease, defined by pleural and subpleural parenchymal fibrosis with deposition of elastic fibers. Median age is reported to be 57 years and, the youngest patient was 24 years old ${ }^{5}$ Hence, our case is the youngest patient reported in the literature. Although the number of cases are increasing in literature, the etiology of IPPFE remains unclear. History of malignancy was found to be $32 \%$ in IPPFE. ${ }^{6}$ Chemotherapy and radiotherapy is also associated with IPPFE. Bone marrow and lung transplantation may cause IPPFE, as a lung manifestation of chronic graft versus host disease. Our patient did not have any history of chemotherapy, radiotherapy or malignancy. Reddy et al. reported recurrent infections in half of patients, Mycobacterium avium-intracellulare and Aspergillus are the most frequent agents. ${ }^{5}$ Genetic and autoimmune factors may also be involved in pathogenesis. IPPFE was reported in siblings in two reports suggesting genetic predisposition as a possible pathogenetic factor. ${ }^{3,7}$ Ancylosing spondylitis, ulcerative colitis, psoriasis with high levels of autoantibodies have been reported as associated conditions. ${ }^{4}$

The most common clinical symptoms of IPPFE are cough, exertional dyspnea, weight loss, which are characteristic for interstitial disease. IPPFE is usually associated with lower body mass index; In IPPFE, lung functions reveal a restrictive lung pattern, with high RV/TLC. Hypoxaemia and hypercapnia may also be seen. Chest radiographs demonstrate bilateral apical pleural thickening, apical volume loss, elevation with hilar opacities and a narrowed anterior-posterior thoracic dimension. Pneumothorax may occur in $\% 30$ of patients and complicates the course of the disease. ${ }^{4,8}$ Our patient had also unilateral pneumothorax. Chest CT shows pleural and subpleural reticular consolidations with interlobular septal thickening of the pleura predominantly at the upper lobes. Structural distortion, traction bronchiectasis and honeycombing may occur. Surgical lung biopsy is essential for the diagnosis. ${ }^{4}$ The histological features include markedly thickened visceral pleura and prominent subpleural fibrosis characterized by elastic fiber deposition in the subpleural area and adjacent pulmonary parenchyma. ${ }^{9}$ Thoracoscopic lung biopsy in our patient revealed subpleural fibrosis and elastic staining demonstrated elastic fiber deposition in the subpleural area and adjacent pulmonary parenchyma. IPPFE is often slowly progressive and refractory to corticosteroids and other immunosuppressive agents. Supportive care and lung transplantation in advanced disease are main therapeutical options. Regarding the mechanisms of elastosis; an antifibrotic drug pirfenidone, may be beneficial for IPPFE. ${ }^{10}$ Pirfenidone inhibits fibrotic factors, especially transforming growth factor-beta. Altough there is no evidence that pirfenidone is effective in IPPFE, Sato et al. reported a case in whom a decline in the pulmonary function was successfully prevented by pirfenidone. ${ }^{11}$

In summary, IPPFE is a rare disorder characterized by predominantly upper lobe fibrosis and mainly occurs in adulthood. To our knowledge, we present the first pediatric case. Therefore, pediatricians should be aware of this disease for the diagnosis and appropriate management.

\section{REFERENCES}

1. Travis WD, Costabel U, Hansell DM, et al. An official American Thoracic Society / European Respiratory Society statement: update of the international multidisciplinary classification of the idiopathic interstitial pneumonias. Am J Respir Crit Care Med 2013;188(6):733-48.

2. Amitani R, Niimi A, Kuze F. Idiopathic pulmonary upper lobe fibrosis. Kokyu 1992;11:693-9.

3. Frankel SK, Cool CD, Lynch DA, et al. Idiopathic Pleuroparenchymal Fibroelastosis: description of a novel clinicopathologic entity. Chest 2004;126(6):2007-13.

4. Watanabe K. Pleuroparenchymal fibroelastosis: Its clinical characteristics. Curr Respir Med Rev. 2013;9:229-37.

5. ReddyTL,TominagaM,HansellDM,etal.Pleuroparenchymal fibroelastosis: a spectrum of histopathological and imaging phenotypes. Eur Respir J. 2012;40(2):377-85.

6. Kusagaya H, Nakamura Y, Kono M, et al. Idiopathic pleuroparenchymal fibroelastosis: consideration of a clinicopathological entity in a series of Japanese patients. BMC Pulm Med 2012;12:72.

7. Azoulay E, Paugam B, Heymann MF, et al. Familial extensive idiopathic bilateral pleural fibrosis. Eur Respir J. 1999;14(4):971-3.

8. English JC, Mayo JR, Levy R, et al. Pleuroparenchymal Fibroelastosis: a rare interstitial lung disease. Respirol Case Rep. 2015;3(2):82-4.

9. von der Thüsen JH. Pleuroparenchymal Fibroelastosis: Its pathological characteristics. Curr Respir Med Rev. 2013;9(4):238-47.

10. BeckerCD, GilJ, Padilla ML. Idiopathic pleuroparenchymal fibroelastosis: an unrecognized or misdiagnosed entity? Mod Pathol. 2008;21(6):784-7.

11. Sato S, Hanibuchi M, Takayashi M, et al. A patient with Idiopathic pleuroparanchymal Fibroelastosis Showing a Sustained Pulmonary Function due to Treatment with Pirfenidone. Intern Med 2016;55(5):497-501. 